\title{
Morfogenesis y dinamica de las vertientes submarinas en la costa de fiordos de Norpatagonia - Chile
}

\author{
Rosemary Vieira \\ Universidad Federal de Río Grande do Sul, Brasil \\ rosemary.vieira@ufrgs.br
}

Recibido 31 julio 2002; versión corregida aceptada, 4 octubre 2002.

\begin{abstract}
RESUMEN
Se estudia los fenómenos de vertientes submarinas y su relación con el fondo de las cubetas en fiordos y canales de Norpatagonia, mediante análisis de registros de perfilador de subfondo. Se observa relación entre la estructura de fondos de cubeta y la dinámica de las vertientes, en dos fases morfogenéticas. La inferior consiste en movimientos en masa subsuperficiales y con características acústicas propias; muestran actividad junto a la base de la vertiente, involucrando materiales aparentemente sin cohesión, posiblemente asociados con flujos de detritos. La superior produjo estructuras superficiales estratificadas de fondo de cubeta; muestra deformaciones por deslizamiento en las vertientes. Rasgos asociados son las depresiones compresionales y sedimentos remoldeados, formados próximos a la base de las vertientes más suaves. Los plunge pools también se ven junto a bases de vertientes, pero en pendientes más elevadas y profundidades mayores. Se observa en los deslizamientos un desplazamiento de la masa sedimentaria más allá de la base de vertiente. Como en vertientes submarinas de las plataformas continentales, y en algunos fiordos, en la zona de canales y fiordos de Norpatagonia, la mayoría de los fenómenos de vertientes se dan más efectivamente en pendientes con menos de $10^{\circ}$. El empleo futuro de técnicas generadoras de datos morfométricos permitirá el análisis más profundo de la dinámica de vertientes y de procesos en distintos ambientes sedimentarios marinos.
\end{abstract}

Palabras clave: geomorfología submarina, fiordo, vertientes submarinas, movimientos en masa, depresiones compresionales, plunge pools.

\section{Submarine slopes morphogenesis and dynamics of Northern Patagonia fjord coast - Chile}

\begin{abstract}
The features of the Northern Patagonia submarine slopes and their relation with the basin bottoms in fjords and channels studied, by means of subbottom profiler records. Two morphogenetic phases show relationships between slope mass movements and bottom structure. The lower phase is represented by distinct acoustic structures, interpreted as possible debris flows of cohesionless material reaching as far as the slope base.The upper phase produced surface stratified sediments; it shows deformity processes produced by slope slides, which can reach far off the slope base. Resulting landforms of such processes are known as compressional depression and remoulded sediments, which has been developed at the lower slope angles. The plunge pools are observed at the slope base, but where the slope is steeper and deeper. As it has been verified in the shelf slopes, and in some basins fjords too, the majority of slope activities occur, effectively, on slopes less than $10^{\circ}$. The future employment of new technologies will be able to provide a important insight into landslide dynamics and process in different submarine sedimentary environment.
\end{abstract}

Key words: submarine geomorphology, fjord, submarines slopes, mass movements, compressional depression, plunge pool. 


\section{INTRODUCCIÓN}

Los movimientos en masa son importantes procesos erosionales y deposicionales, y son un efectivo mecanismo que actúa sobre la forma y el desplazamiento de grandes cantidades de sedimentos en ambientes submarinos (McADOO et al. 2001; McADOO, 2000a y b). Sin embargo, la gran mayoría de estudios se ha concentrado más en áreas de la plataforma continental que en los fiordos, debido a las actividades económicas que se desarrollan en las primeras, como la petrolífera.

Considerando las formas y procesos de las vertientes submarinas, los fiordos son un ambiente ideal para el estudio de casi todas las formas de depósitos de movimientos de masa en función de la existencia de vertientes con pendientes muy fuertes, y por las tasas de sedimentación que en muchos casos superan las tasas de consolidación (SYVITSKI, et al. 1987).

En Chile, desde el punto de vista de la morfología submarina, de la morfogénesis y procesos de los fondos y de las vertientes de los fiordos en Chile, los primeros estudios han sido desarrollados por ARAYA - VERGARA (1996, 1997, 1998, 1999a, b, 2000), teniendo como base un gran conjunto de registros de perfilador de subfondo de alta resolución y muestras de sedimentos que han sido recolectados durante los últimos seis años, a través de los cruceros de investigación efectuados por la Armada de Chile y por el Comité Oceanográfico Nacional - Cruceros CimarFiordo; de datos cronológicos generados por el mismo crucero (SALAMANCA, 1996) y por testigos del crucero R/V Polar Duke PD93-06 (LEVENTER et al. 1995).

En esos estudios, ARAYA - VERGARA $(1996,1997)$ identifica los principales ambientes morfogenéticos submarinos, clasificando dos tipos de cuencas de fiordos: con explanadas de represamiento, y con explanadas de represamiento alternadas con acu- mulaciones caóticas (ARAYA - VERGARA, 1998). La discusión avanza más hacia al sur, con la identificación de las formas submarinas principales de los fiordos de Patagonia Central y su confrontación con las formas del Würm y del Holoceno en el medio subaéreo de la región (ARAYA - VERGARA, 1999b); con el establecimiento de una secuencia espacio-temporal de las formas deposicionales del fondo de la fractura longitudinal de Patagonia Central (ARAYA - VERGARA, 1999a), y finalmente, con la morfogénesis de los piedmonts submarinos reconocidos en el Estrecho de Magallanes y Bahía Nassau, en Chile Austral, tomando como referencia la posición de morrenas subaéreas de la región (ARAYA - VERGARA, 2000).

Pese la importancia de las vertientes en la distribución de los sedimentos en los fiordos, y del valor geocronológico de los procesos genéticos que involucran su arquitectura, esa clase de investigación solamente ha sido desarrollada en Chile por ARAYA - VERGARA (1996; 1997; 1998; 1999a, b, 2000).

Considerando la importancia de los inéditos datos originados por los cruceros CIMAR FIORDO en la costa patagónica y de los estudios iniciados por ARAYA - VERGARA (1996, 1997, 1998, 1999a, b, 2000), este trabajo tiene el propósito de avanzar en la sistemática sobre la morfogénesis, dinámica y evolución de vertientes submarinas en fiordos y canales de Norpatagonia; desde el punto de vista de los movimientos en masa y sus relaciones con la estructuración de fondos.

\section{MATERIALES Y MÉTODOS}

\section{Area de estudio}

El área de estudio comprende la costa de fiordos de sur de Norpatagonia, que incluye los fiordos y canales indicados en la Fig. 1. El significado de esta región y las características de las unidades estudiadas han sido mostradas por ARAYA-VERGARA (1996, 1997 y 1998). 


\section{ÁREA DE ESTUDIO}

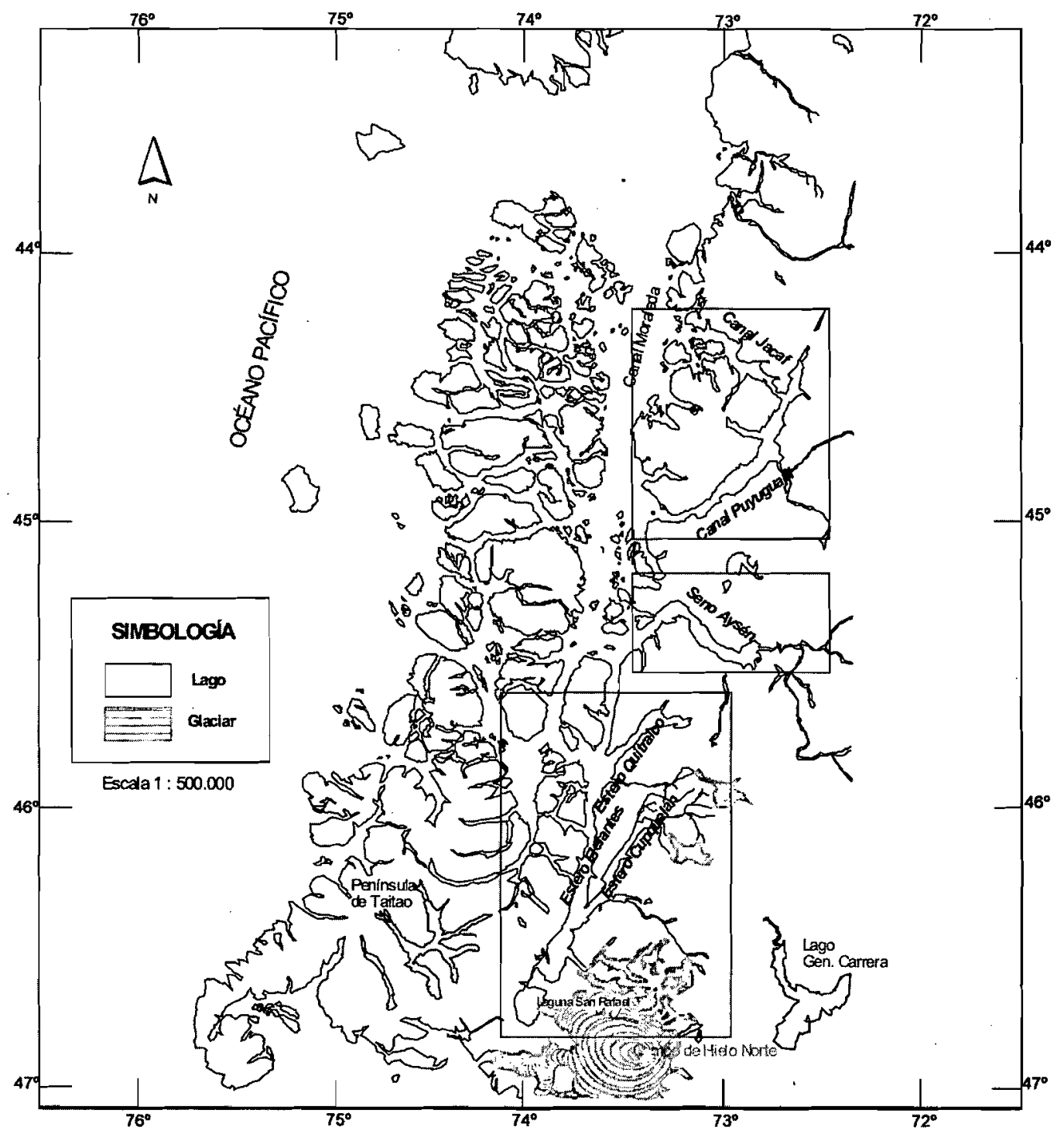

Fig. 1. Área de estudio

Fig. 1: Area of study 

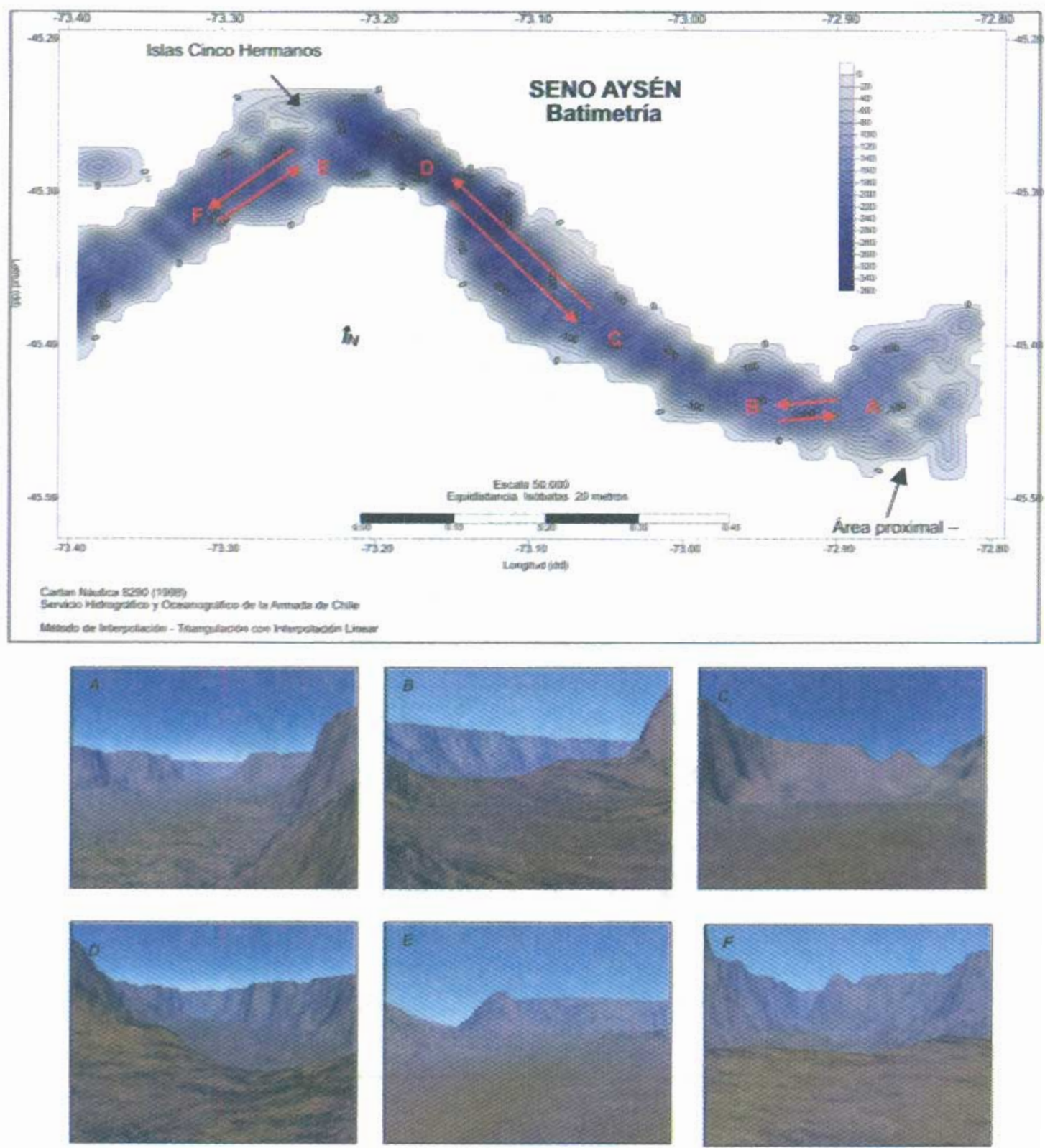

Fig. 2: Fiordo Aysén. Mapa batimétrico y modelos 3D de la topografía del fíordo Fig. 2: Aysén Fjord. Bathymetric map and 3D models of fjord topography 


\section{Adquisicion de datos y procesamiento}

Registros de alta resolución $(3.5 \mathrm{kHz})$

La observación de la morfología de fondo y de los procesos de vertientes se hizo mediante registros de perfilador de subfondo Edo Western, modelo $248 \mathrm{E}$, recolectados a $3.5 \mathrm{kHz}$ durante el crucero CIMAR - FIORDO I, realizado en 1995 en el Agor Vidal Gormaz y por iniciativa del Comité Oceanográfico Nacional. Por razones logísticas, los registros acústicos cubren la mayor parte de los perfiles longitudinales de cada fiordo, no transversales. Para interpretar las superficies en términos de la naturaleza de los materiales, se consideró que el sonar de $3.5 \mathrm{kHz}$ posee gran variabilidad de penetración desde decenas de metros en sustratos fangosos blandos, hasta unos pocos metros en sustratos arenosos (CARLSON, 1989).

La interpretación de los caracteres acústicos se basó en la clasificación de DAMUTH (1978) y en las experiencias de KARL (1989), SYVITSKI \& PRAEG (1989), ABARRUZA (1991), CAI et al (1997), DasILVA et al. (1997), SYVISTSKI \& LEE (1997), GILBERT et al. (1998), PRIETO et al. (1999), TAYLOR et al. (2000), BØE et al. (2000) y ARAYÄ - VÉRGARA (1997, 1998, 1999a y b, 2000). Se consideró los siguientes rasgos del registro: (i) grado de intensidad de las superficies reflectoras; (ii) la configuración de la reflexión, clasificada en estrațificada, caótica y transparente y (iii) la geometría externa (STOKER et al. 1997).

\section{Escala vertical}

Los̀ registros acústicos fueron obtenidos a escalas variables, calculadas a partir de la velocidad del buque, de los tiempos anotados y de las coordenadas GPS. Los registros fueron obtenidos a bordo escalas aproximadas 1:375, 1:750 6 1:1.500. Así, la exageración vertical de la escala fluctúa entre 10 y 50 , lo que permite una mayor ampliación y distorsión de los rasgos morfológicos.
Clasificación de los movimientos en masa

La identificación de los principales procesos de movimientos en masa y sus depósitos correspondientes se basó en los siguientes sistemas:

- la clasificación de los flujos de sedimentos por gravedad en offshore de MIDLETON \& HAMPTON (1979, in KENETT, 1982) y de MULDER \& COCHONAT (1996);

- la clasificación adaptada de SYVITSKI et al. (1987) y de KOSTASCHUK \& McCANN (1987) para la interpretación de vertientes submarinas de deltas en fiordos;

- la clasificación para depósitos de sedimentos por flujo de gravedad basadas en las condiciones de flujo durante la sedimentación de POSTMA (1986).

- la distribución de mecanismos en los deslizamientos en las vertientes fue basada en el Multilingual Landslide Glossary, de International Geotechnical Societies, UNESCO Working Party for World Landslide Inventory (1993), reproducido en DIKAU et al. (1996).

\section{RESULTADOS}

\section{Morfologia general del perfil longitudinal}

Los fiordos de orientación SW - NE, Cupquelán, Quitralco y Puyuguapi, cuyas partes distales se comunican con el fiordo Elefantes y canal Moraleda, respectivamente, tienen el fondo transversalmente cóncavo. Sus cuencas son estructuradas en cubetas separadas por umbrales de naturaleza rocosa. Las cubetas entre umbrales sustentan formas deposicionales en estructura pool (Fig. $4 b)$.

Los cuerpos de sedimentos de los dos primeros fiordos se estructuran de forma lamina$\mathrm{da}$, con multicapas horizontales de espesores variables, representadas por reflectores acústicos fuertes medios y débiles, y sumando potencias visibles entre 30 a 40 metros. La profundidad media de la cubeta del fiordo Cupquelán es de $200 \mathrm{~m}$, mientras del fiordo Quitralco es de 150 (Fig. 4b). Los dos fiordos poseen extensiones no inferiores a $30 \mathrm{~km}$. El 


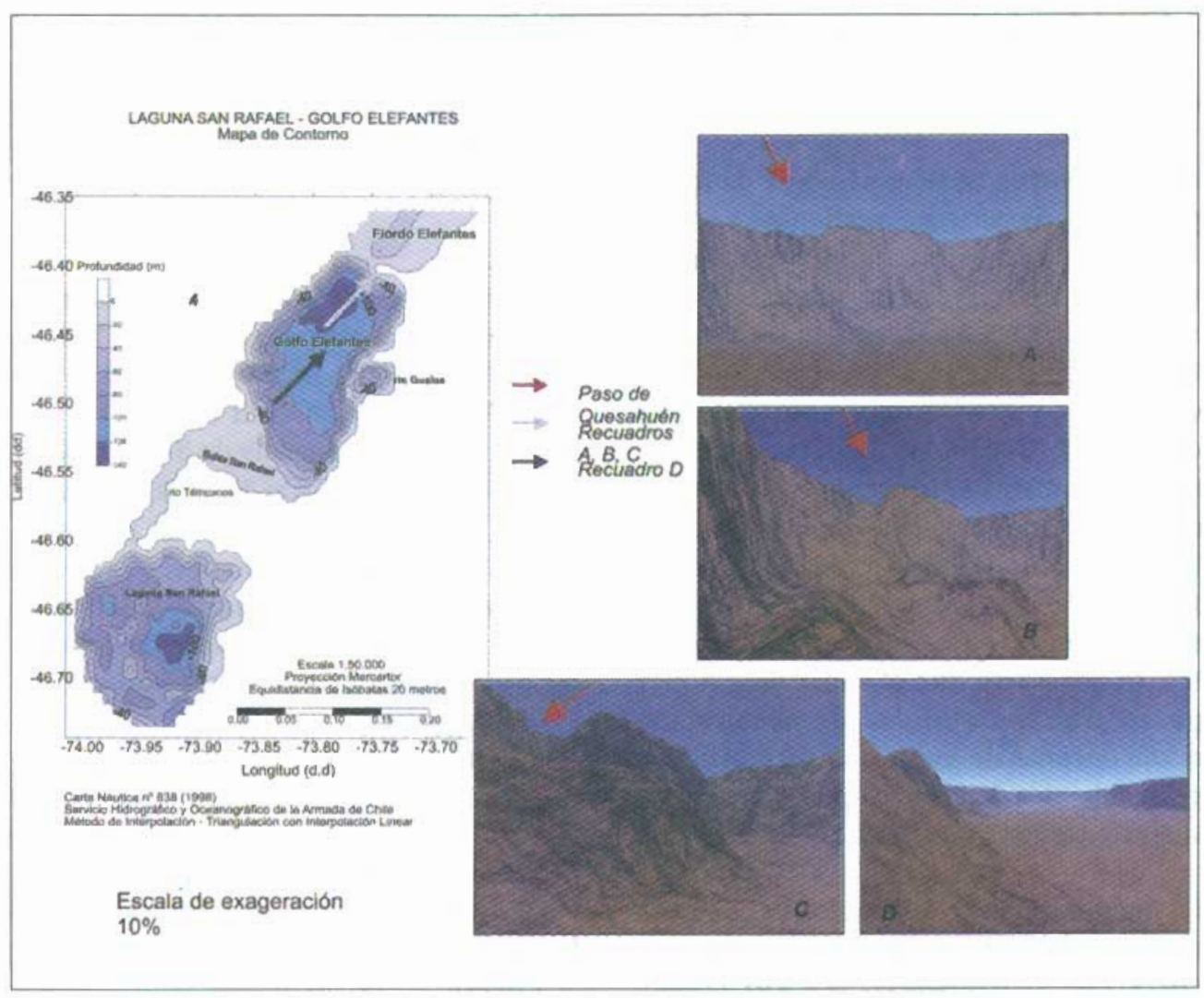

Fig. 3: Golfo Elefantes. Mapa batimétrico y modelos 3D de la topografía del fiordo

\section{Fig. 3: Elefante Golf. Bathymetric map and 3D models of fjord topography}

fiordo Puyuguapi se extiende por más de 80 $\mathrm{km}$. En su fondo, las capas sedimentarias suman potencias visibles entre 20 y 25 metros.

El canal Jacaf y el fiordo Aysén se caracterizan por la irregularidad del fondo y por la existencia de profundas cubetas.

En el canal Jacaf, con cerca de 50 kilómetros de largo, tres aspectos caracterizan la morfología submarina: (i) la gran irregularidad de su fondo; (ii) la gran cantidad y la altura de los umbrales; $y$ (iii) la presencia de depresiones intra - umbrales rellenadas por espesas capas sedimentarias. Algunos de los umbrales acompañan formas subaéreas, como lo que se verifica en el cordón de islas en la parte noroeste del canal, en la confluencia con el canal Moraleda. Se nota, además, que las subcuencas internas del canal Jacaf tienen profundidades superiores a de los canales vecinos, alcanzando algunas más de $650 \mathrm{~m}$. Las cubetas más profundas coinciden con la confluencia con los fiordos tributarios. Las formas deposicionales del canal Jacaf se presentan en tres distintos tipos de emplazamiento: sobre y entre los umbrales más rasos en la parte distal del canal, en el fondo de cubetas y sobre las vertientes de los umbrales, como ya había identificado ARAYA - VERGARA (1997).

El fiordo Aysén, con una longitud de alrededor de $73 \mathrm{~km}$ y una profundidad media de 142 metros (Fig. 2), se caracteriza - en el sector que se extiende desde las Islas Cinco Hermanos hasta la confluencia al canal Moraleda - por la concentración de umbrales y, por con- 

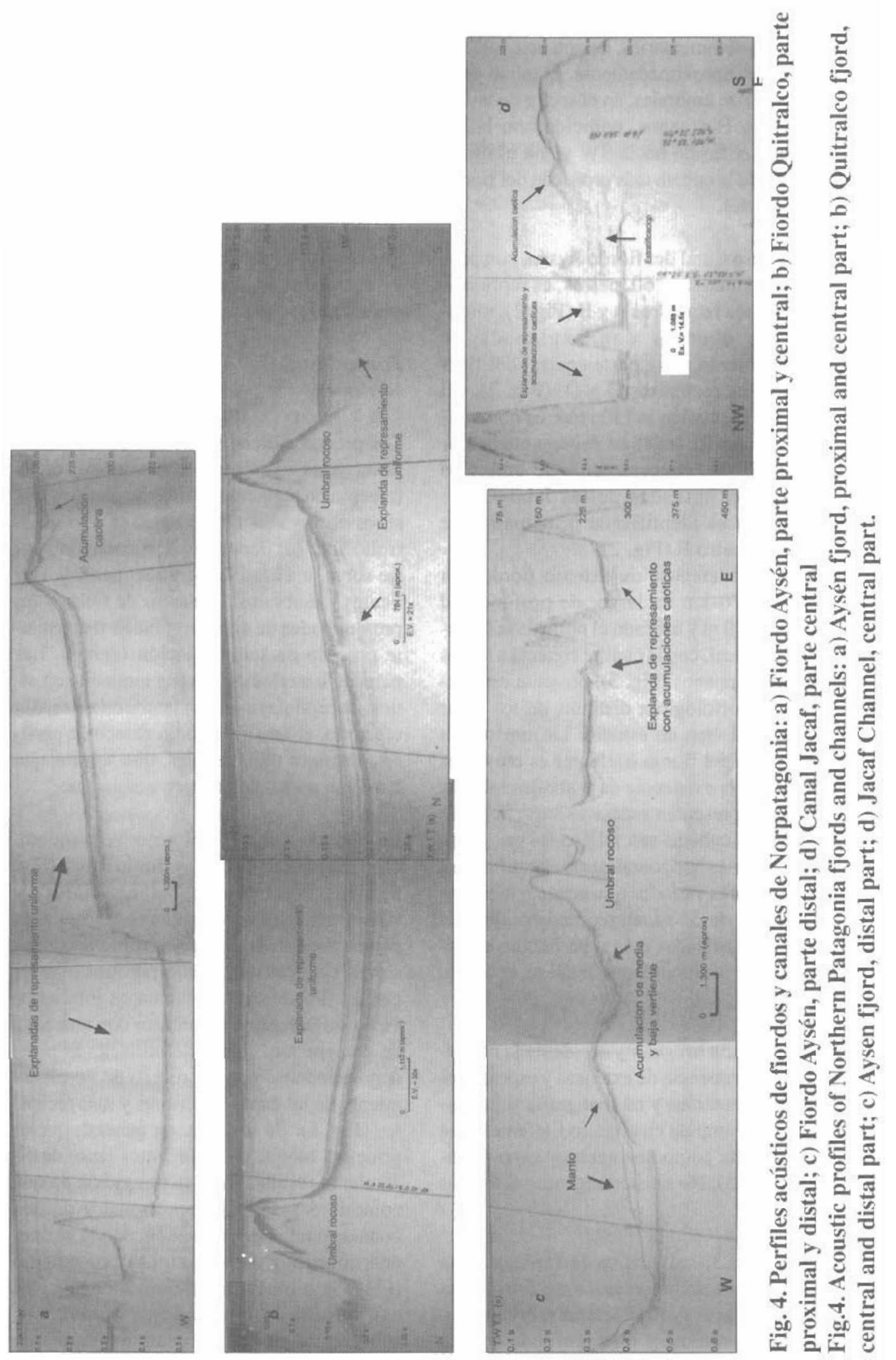
siguiente, de cubetas igualmente rellenadas por capas sedimentarias, de potencia total de 70 metros, aproximadamente. El inicio de la secuencia de umbrales, en el sector de las Islas Cinco Hermanos, coincide con la inflexión del fiordo hacia SW y con el límite externo de la cubeta más profunda del fiordo (Figs. 2, 4c).

El área proximal del fiordo Aysén, con profundidad media de 60 metros, es representada por los recuadros A y B (Fig. 2), mientras las cuencas con explanada de represamiento de la parte central del fiordo, por los recuadros C y D. (Fig. 2). En ellos, se identifica la falla que es observada en el perfil acústico representado por la Fig. 4a. La concentración de umbrales en las proximidades de las Islas Cinco Hermanos es identificada principalmente en el recuadro F (Fig. 2).

El fiordo Elefantes, un extenso fiordo con cerca de $70 \mathrm{~km}$ de largo, de profundidad media de $50 \mathrm{~m}$ y ubicado al norte de la Laguna San Rafael, con la cual se conecta a través del río Témpanos (Fig. 3), presenta características morfológicas distintas de los otros fiordos del área de estudio. La morfología submarina del fiordo Elefantes es muy irregular, con la existencia de umbrales rocosos rasos que presentan sucesivas superficies de fallas. Las cubetas son rellenadas por capas sedimentarias horizontalmente estratificadas, de reflectores variados que tienen una potencia visible de $35 \mathrm{~m}$, aproximadamente. Las capas estratificadas más superficiales se en cuentran a profundidades de $50 \mathrm{~m}$, entre los umbrales.

La topografía irregular y accidentada no permite una secuencia de extensas y espesas capas sedimentarias, y tal topografía solamente es interrumpida en el golfo Elefantes, que se comporta como una ancha y extensa cubeta (Fig. 3). No existen registros acústicos del área.

En la Fig. 3, se verifica la ruptura de la secuencia de umbrales rasos del fiordo Elefantes hacia el golfo Elefantes, donde empieza una extensa y más profunda cuenca con explanada de represamiento (recuadro D). El Paso de Quesahuén, que representa tal hito, es observado por los recuadros $\mathrm{A}$, $\mathrm{B}$ y $\mathrm{C}$ en distintos niveles de acercamiento.

\section{Descripcion y distribucion del carácter acustico}

Las características acústicas de las formas y estructuras deposicionales están esquematizadas en las Tablas 1 y 2 . Formas asociadas a los fenómenos de
vertientes

Los procesos de movimientos en masa más observados incluyen deslizamientos, creep (creeping o reptación) o flujo de detritos. Los sitios observados más propensos a su desarrollo son: (a) donde la sedimentación ocurre sobre una base topográfica marcada vertientes y umbrales); (b) áreas de fallas y (c) proximidades de áreas con fuerte o constante proceso de sedimentación (deltas). Las paredes laterales de fiordos también son sitios susceptibles a movimientos en masa. Sin embargo, el área de estudio carece de perfiles acústicos transversales, que son los que habilitan para analizar esos ambientes.

En algunas cubetas del seno Ventisquero, canal Puyuguapi (Fig. 5) y fiordo Aysén (Fig. 6), los sedimentos superficiales y subsuperficiales se deforman en las cercanías de una zona de compresión, como acumulaciones caóticas recubiertas porotras mảs recientes. Puede ocurrir que capas interiores, ya deformadas por el contàcto con una zona de compresión, sufran deformaciones postdeposicionales, por el proceso de desplazamiento de las capas superiores y más recientes (Fig. 5). Se observa, en general, que el principal bloque de sedimentos sufre deslizamiento resultando en la formación de una zona de depresión compresional y de una zona de punta de compresión, donde se pueden constituir deformaciones por empuje (Figs. 5a y b). En los ejemplos citados, las estructuras laminadas super y subsuperficiales se encuentran siempre en 
Tabla 1: Caracteres acústicos

Table 1: Echo-Characters

\begin{tabular}{|c|c|c|}
\hline & \multicolumn{2}{|c|}{ Caracter del eco Explanadas de fondo de cuenca } \\
\hline FIORDO & Explanada de represamiento uniforme & $\begin{array}{l}\text { Explanada de represamiento y acumulaciones } \\
\text { caóticas }\end{array}$ \\
\hline Jacaf & & $\begin{array}{l}\text { En fondo plano, a veces con suaves ondulaciones; } \\
\text { intensidad moderada del tono; reflectores de } \\
\text { subfondo continuos, estratificados y paralelos, semi } \\
\text { transparentes. }\end{array}$ \\
\hline Ventisquero & . & $\begin{array}{c}\text { En fondo plano, intensidad moderada del tono; } \\
\text { reflectores de subfondo continuo, estratificados, } \\
\text { paralelos e intercalados con reflectores semi - } \\
\text { transparentes. }\end{array}$ \\
\hline Puyuguapi & $\begin{array}{l}\text { En fondo plano, intensidad del tono moderada a } \\
\text { elevada; reflectores de subfondo continuos, } \\
\text { estratificados, paralelo; estructura horizontal. }\end{array}$ & \\
\hline Aysén & $\begin{array}{l}\text { En fondo plano, intensidad del tono moderada a } \\
\text { elevada; reflectores de subfondo continuos; poco } \\
\text { estratificados; sobreyace a una masa no continua, } \\
\text { acercándose a una configuración caótica. } \\
\text { En fondo con suave pendiente, intensidad del } \\
\text { tono moderada; reflectores de subfondo } \\
\text { continuos, estratificados; intercalados con } \\
\text { reflectores transparentes; reflectores más internos } \\
\text { se muestran acuñados en algunos sectores }\end{array}$ & $\begin{array}{c}\text { En fondo ondulado y con suaves pendientes, } \\
\text { intensidad moderada a elevada; reflectores de } \\
\text { subfondo continuos a semicontinuos; estratificado, } \\
\text { intercalados con reflectores semi - transparentes; } \\
\text { sobreyacen a reflectores opacos y de configuración } \\
\text { cabtica. }\end{array}$ \\
\hline Quitralco & $\begin{array}{l}\text { En fondo relativamente cóncavo, intensidad del } \\
\text { tono moderada a elevada; reflectores de subfondo } \\
\text { continuos, estratificados; intercalados con } \\
\text { reflectores transparentes; sobreyacen a } \\
\text { reflectores continuos de elevado tono. Más al } \\
\text { interior se encuentran reflectores de carácter no } \\
\text { continuo, semi - estratificado. } \\
\text { En fondo plano, intensidad elevada del tono; } \\
\text { reflectores de subfondo semi continuos; } \\
\text { sobreyacen a masa opaca y de configuración } \\
\text { caótica. }\end{array}$ & \\
\hline Cupquelán & $\begin{array}{c}\text { En fondo plano, intensidad muy elevada del tono } \\
\text { reflectores de subfondo continuos, estratificados; } \\
\text { sobreyacen a reflectores no continuos y semi- } \\
\text { transparentes }\end{array}$ & \\
\hline Elefantes & $\begin{array}{c}\text { En fondo plano, intensidad muy elevada del tono; } \\
\text { reflectores de subfondo semi - continuo, semi - } \\
\text { estratificado y semi transparente; se intercala con } \\
\text { reflectores continuos. }\end{array}$ & . \\
\hline
\end{tabular}


Tabla 2: Caracteres acústicos

TABLE 2: EChO-CHARACTERS

\begin{tabular}{|c|c|c|}
\hline & \multicolumn{2}{|c|}{ Caracter del eco } \\
\hline FIORDO & Umbrales & Vertientes medias y base de vertientes \\
\hline Jacaf & $\begin{array}{l}\text { Distal - Intensidad del tono moderada a intensa; } \\
\text { reflectores internos transparentes; hipérbolas } \\
\text { amplias e irregulares con variadas elevaciones de } \\
\text { los vértices } \\
\text { Central - Intensidad del tono moderada a } \\
\text { elevada; reflectores internos transparentes; } \\
\text { hipérbolas amplias e irregulares; elevados } \\
\text { vértices. } \\
\text { - } \quad \text { Intensidad del tono moderada a débil; } \\
\text { reflectores internos opacos y de } \\
\text { configuración caótica; hipérboles } \\
\text { amplias. } \\
\text { Intensidad del tono moderada a débil; } \\
\text { reflectores internos opacos y } \\
\text { configuración caótica; forma de bloques. }\end{array}$ & $\begin{array}{l}\text { Distal - Tono moderado; reflector de subfondo } \\
\text { semi - transparente a transparente; forma externa } \\
\text { ondulada y a veces acuñada, cumbre con algunos } \\
\text { vértices. } \\
\text { Central - Tono moderado a débil; reflectores } \\
\text { internos opacos y de configuración caótica. } \\
\text { Intensidad débil; reflectores internos semi - } \\
\text { estratificados intercalados con reflectores caóticos }\end{array}$ \\
\hline Ventisquero & $\begin{array}{l}\text { Intensidad elevada del tono; reflectores internos } \\
\text { transparentes; hipérbolas largas e irregulares con } \\
\text { variadas elevaciones de los vértices. } \\
\text { Intensidad moderada a débil del tono; reflectores } \\
\text { internos opacos y configuración caótica; } \\
\text { hipérbolas amplias. }\end{array}$ & $\begin{array}{l}\text { Intensidad elevada del tono, reflectores internos } \\
\text { semi - transparentes; formas acuñadas. }\end{array}$ \\
\hline Puyuguapi & $\begin{array}{l}\text { Central - Intensidad moderada a elevada del } \\
\text { tono; reflectores internos transparentes; forma } \\
\text { única hiperbólica. }\end{array}$ & $\begin{array}{l}\text { Intensidad elevada del tono; reflectores internos } \\
\text { semi - transparentes; estratificada con } \\
\text { deformaciones; sobreyace a masa interna opaca y } \\
\text { caótica. }\end{array}$ \\
\hline Aysén & $\begin{array}{l}\text { Distal - Intensidad del tono moderada a } \\
\text { elevada, reflectores internos transparentes; } \\
\text { forma irregulares con puntas aisladas. } \\
\text { Central - Intensidad del tono moderado; } \\
\text { reflectores internos transparentes; formas únicas } \\
\text { hiperbólicas. } \\
\text { Proximal - Intensidad moderada a elevada del } \\
\text { tono; reflector interno opaco y caótico; } \\
\text { microhiperbólicas (hummocky). }\end{array}$ & $\begin{array}{l}\text { Distal - Intensidad del tono moderada; reflectores } \\
\text { internos opacos; algunas vertientes cubiertas por } \\
\text { mantos de reflectores internos semi - transparentes } \\
\text { a transparentes. } \\
\text { Central - Intensidad del tono moderada; reflectores } \\
\text { internos opacos y caóticos; forma acuñada y } \\
\text { sobreyacen parte de las formas de explanada del } \\
\text { fondo de cuenca. } \\
\text { Proximal - Intensidad moderada a elevada del } \\
\text { tono; reflectores internos semi - transparentes, } \\
\text { estratificación hacia la base de la vertiente. }\end{array}$ \\
\hline Quitralco & $\begin{array}{l}\text { Intensidad del tono elevada; reflectores internos } \\
\text { transparentes; hipérbolas amplias e irregulares. }\end{array}$ & $\begin{array}{l}\text { Intensidad elevada del tono; reflectores internos } \\
\text { opacos; deformaciones proximales en algunas } \\
\text { bases de vertiente y acumulaciones sucesivas en } \\
\text { forma de cuñas en otras. }\end{array}$ \\
\hline Cupquelán & $\begin{array}{l}\text { Distal - Intensidad elevada del tono; reflectores } \\
\text { internos transparentes; hipérbolas amplias e } \\
\text { irregulares. }\end{array}$ & $\begin{array}{l}\text { Distal - Intensidad elevada del tono; reflectores } \\
\text { internos estratificados y continuos; sobreyacen a } \\
\text { masa opaca y caótica que se prolonga hasta la } \\
\text { base; concentración de acumulaciones con } \\
\text { reflectores internos transparentes. }\end{array}$ \\
\hline Elefantes & $\begin{array}{l}\text { Intensidad elevada del tono; reflectores internos } \\
\text { transparentes; hipérbolas amplias e irregulares; } \\
\text { extensos umbrales con sectores de concentración } \\
\text { de hipérbolas. }\end{array}$ & $\begin{array}{l}\text { Intensidad elevada del tono; reflectores internos } \\
\text { opacos y caóticos. }\end{array}$ \\
\hline
\end{tabular}



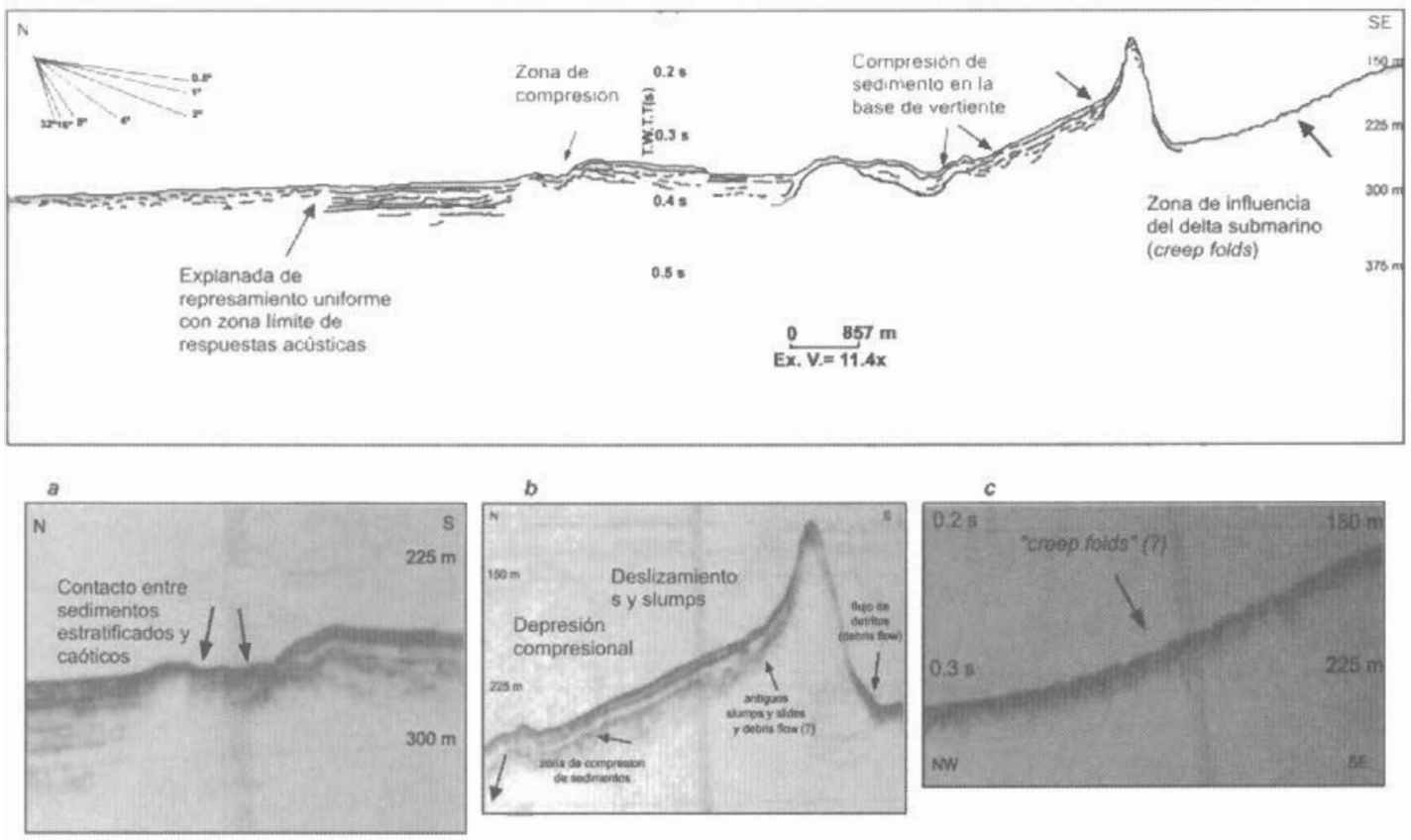

Fig. 5. Fiordo Puyuguapi. Movimientos en masa se reproducen en vertientes con pendientes entre $1-8^{\circ}$. Acumulaciones caóticas internas sirven como zonas de compresión para sedimentos superiores de carácter estratificado, que sufren deformaciones en superficie y en subsuperficie, algunas en forma de cuña (a). En la base de la vertiente una depresión compresional se forma antes de la acumulación de sedimento remoldeado. Sobre el umbral de origen rocoso se desprenden materiales que se acumulan en la base de las vertientes, formando una zona de compresión de sedimentos (b). Hacia el área de delta submarino se notan formas suavemente dobladas, concentradas vertiente abajo, que se asocian probablemente a movimientos de reptación (creep), que se desarrollan en pendientes de $2^{\circ}$ (c).

Fig. 5: Puyuguapi fjord. Mass movements at slopes of $1-8^{\circ}$. Internal chaotic accumulations act as compressional zones for upper stratified sediments, which undergo at surface and subsurface, some with wedge shape (a). At the slope toe, a compressional depression in front of remoulded sediment accumulation. Over the rocky sill material moves slope down with accumulation at its toe, producing a compression sediments zone (b). At the submarine fan area, gently folded landforms are observed, concentred slope down, and which are probably associated with creep movements at slops of $2^{\circ}(\mathrm{c})$. 
fase superior a las estructuras caóticas y de carácter acústico semi-transparente.

En cuencas de fiordos con explanadas de represamiento uniforme, se ve deformaciones de las capas laminadas desde los bordes de las cubetas y avanzando, en algunos ejemplos, hasta las proximidades del otro límite de la cubeta (Fig.5).

En algunas cubetas del seno Ventisquero, canal Puyuguapi (Fig. 5) y fiordo Aysén (Fig. 7), los sedimentos superficiales y subsuperficiales se deforman en las cercanías de una zona de compresión, como acumulaciones caóticas recubiertas. Puede ocurrir que capas interiores, ya deformadas por el con-

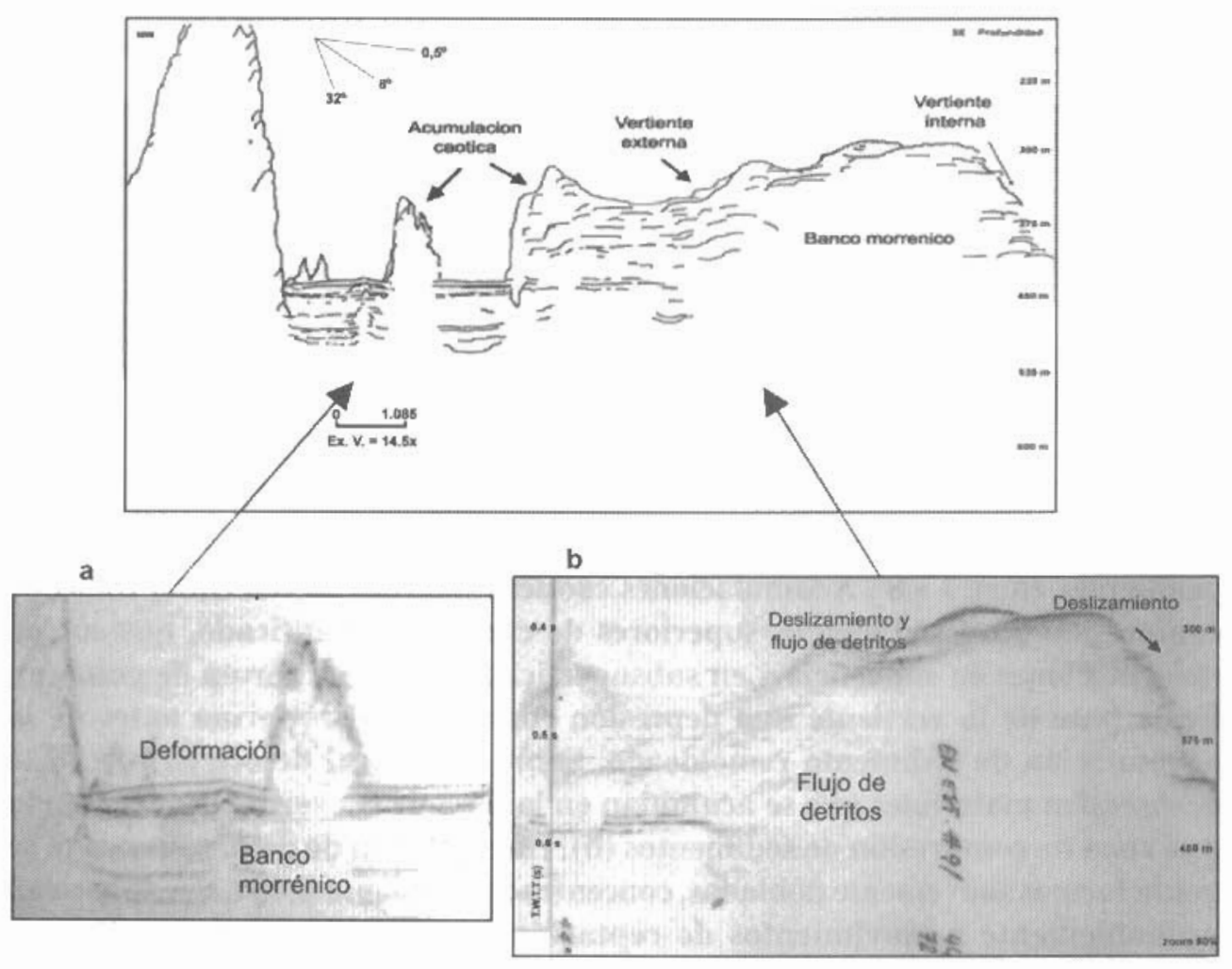

Fig. 6. Canal Jacaf. Cuencas con explanada de represamiento y acumulación caótica, donde se observa un posible banco morrénico, cuya vertiente interna tiene pendiente más elevada que la externa. En esa última se realizan movimientos en masa, como deslizamientos y flujo de detritos que se engranan rítmicamente con depósitos de estructura laminada (a y b). En los sedimentos represados se nota deformación de los sedimentos por compresión (a).

Fig. 6. Jacaf channel. Basins with uniform ponding esplanade and chaotic accumulation, where a morrenic banc is observed, which internal slope is steeper than the external one. In the last one, landmass movement are executed, as slides and debris flows, whose deposits are rhythmically inter-stratified with laminated beds ( $\mathrm{a} \mathrm{y}$ b). The ponded sediments are deformed by compression. 


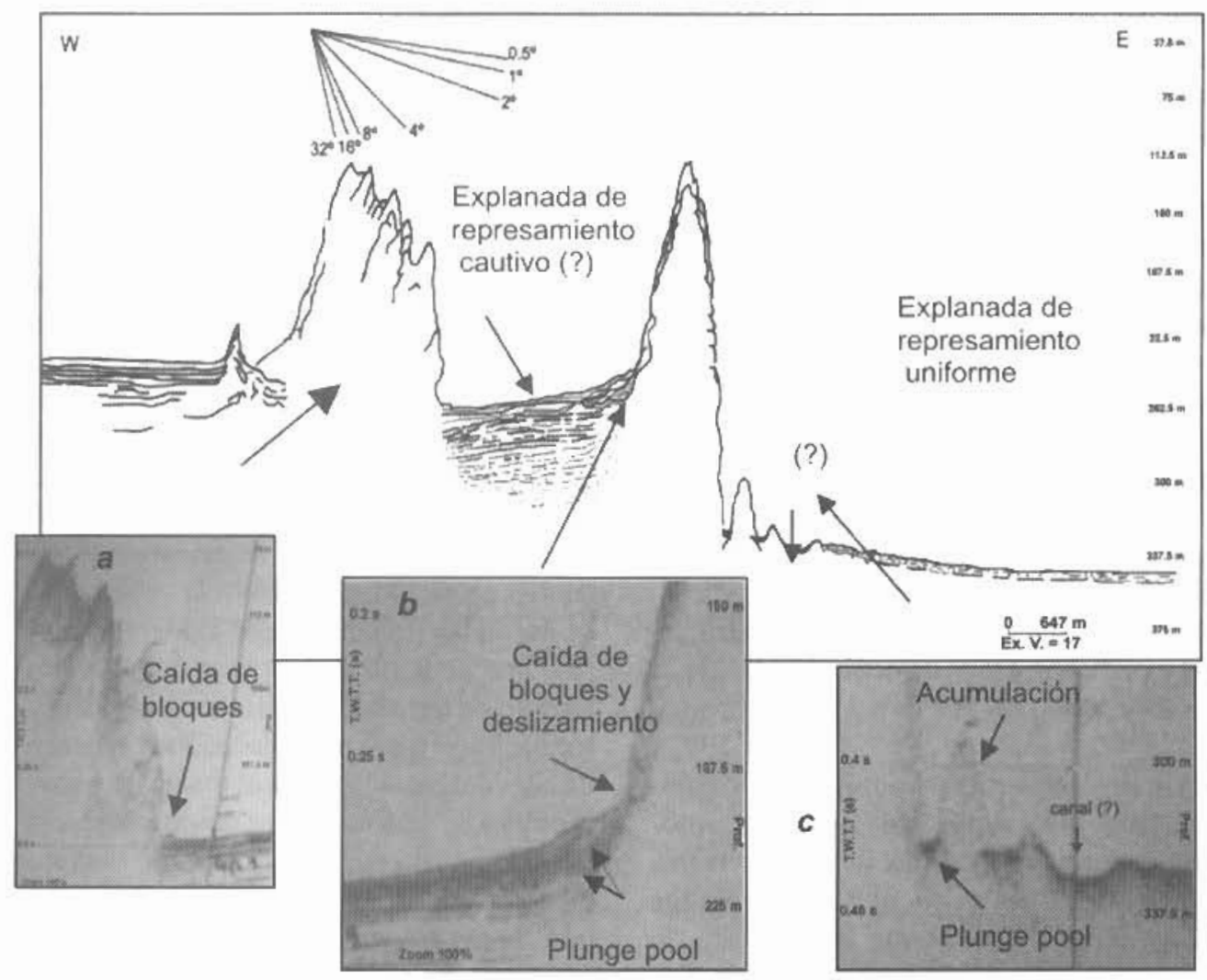

Fig. 7. Fiordo Aysén. Sector Isla Cinco Hermanos. Pendientes más elevadas favorecen el deslizamiento de bloques hacia la base de la vertiente (a), estando en algunas cubetas, en pendientes de $2^{\circ}$ o más (b). Es posible visualizar depresiones plunge pool junto a la base de las vertientes con pendientes superiores a $32^{\circ}$ (b y c).

Fig. 7. Aysén fjord. Isla Cinco Hermanos sector. Steeper slopes allow the slides through the toe (a), some of then at gradient of $2^{\circ}$ or more (b). It is possible to visualize plunge pool next to the toe of the slopes with gradients upper $32^{\circ}$.

tacto con una zona de compresión, sufran deformaciones post-sedimentarias, por el proceso de desplazamiento de las capas superiores y más recientes (Fig.5). Se observa en general, que el principal bloque de sedimentos sufre deslizamiento resultando en la formación de una zona de depresión compresional y de una zona de punta de compresión, donde se pueden formar varias formas dobladas por empuje (Figs. 5 a y b). En los ejemplos citados, las estructuras laminadas super y subsuperficiales se encuentran siempre en fase superior a las estructuras caó- ticas y de carácter acústico semi-transparente.

En el fiordo Puyuguapi, la deformación en forma de pliegues de la estructura sedimentaria asociada con la fuerte intensidad del tono del fondo y la ausencia de respuesta del subfondo próximo a la zona de desembocadura del río Cisnes, observadas aún en la Fig. $5 c$, indican un probable ejemplo de reptación (creep o creeping). Aunque la reptación sea un fenómeno de vertiente típicamente subaéreo, esa clase de deforma- 
ción del sedimento es considerado ubícua en la mayoría de los fiordos con elevada sedimentación o en vertientes más abruptas de áreas de prodeltas.

En el fiordo Aysén, una serie de cubetas más profundas en distintos niveles son separadas por umbrales aparentemente rocosos, con pendientes superiores a $30^{\circ}$. El relleno de esas cubetas probablemente se efectuó por deposición de sedimentos glacimarinos (durante el periodo de retroceso glacial) y por sedimentos marinos más recientes (bypass) represados por los grandes umbrales. La acumulación de sedimentos en la base de vertientes con pendientes más elevadas proporciona deslizamientos de las capas superficiales y probables flujos de detritos en las capas inferiores.

En la base de vertientes abruptas de los umbrales rocosos, rupturas de sedimentos producen formas separadas de las vertientes por depresiones conocidas como plunge pools (Fig. 7). Hay que diferenciar, sin embargo, entre plunge pools y depresiones compresionales; los primeros se desarrollan en la base de vertientes con pendientes más fuertes, mientras las formas subsiguientes están asociadas a pendientes más suaves.

En cuencas con explanadas de represamiento y acumulaciones caóticas como las identificadas en el canal Jacaf y fiordo Aysén, ocurren formas y procesos no identificados en los demás fiordos. En el canal Jacaf, la acumulación de sedimentos caóticos originó estructuras deposicionales en formas ligeramente tabulares, cuyos materiales de la vertiente engranan con capas de estructura laminada, alternadamente. El bloque de sedimentos tiene pendientes externas (hacia $W$ ) de $4^{\circ}$, y pendientes internas sobre $8^{\circ}$ (Fig. 6).

$\mathrm{Al}$ contrario de lo que ha sido verificado en los demás fiordos, en el canal Jacaf, la zona de contacto entre los reflectores internos incoherentes y las capas laminadas de la explanada de represamiento es caracterizada por el engranamiento rítmico de las primeras con las segundas, formando cuñas. Esas formas acuñadas son observadas inicialmente por ARAYA - VERGARA (1998), que las denomina provisoriamente de lenguas de materiales caóticos (Fig.6).

En las áreas distales, tanto del fiordo Aysén como del canal Jacaf, importantes elementos las distinguen de otras secciones del fiordo (Fig. 3). En el primero, se observan dos tipos de deformación de las capas de sedimentos con reflectores acústicamente transparentes: (i) deformación sinsedimentaria en los sedimentos que rellenan las depresiones y que cubren elevaciones, debido a la adaptación de esas a las irregularidades topográficas; y (ii) corte de parte de las capas dándoles un aspecto de terrazas. La primera clase de deformación es igualmente encontrada en áreas distales del canal Jacaf. Esos mantos son susceptibles de deslizamientos desde la vertiente superior. Sin embargo, al mismo tiempo, dichas deformaciones en la capa de manto que cubren las vertientes y rellenan las depresiones en las partes distales de ambos fiordos, presentan rasgos post-sedimentarios (epigénicos), debido a la existencia de sedimentos remoldeados por deslizamientos, además de deformaciones convexas, posiblemente producidas por empuje.

Elementos igualmente notables aparecen en áreas próximas a la desembocadura del fiordo Aysén, donde sedimentos estratificados se distribuyen sobre las dos vertientes de una estructura de caótica sedimentos. La pendiente varía entre 4 y $8^{\circ}$ de acuerdo con la conformación de los estratos. Entre esas dos estructuras y sobre la baja vertiente de la última, se desarrolla una tercera estructura de reflectores internos opacos y de $4^{\circ}$ de pendiente, cuya deformación se nota en su parte superior. Lo mismo se produce en las capas estratificadas superiores. Sobre las vertientes externas, las espesas estructuras laminadas se desplazan hacia la explanada y producen deformación próxima al borde externo de la cubeta (Fig. 8). 


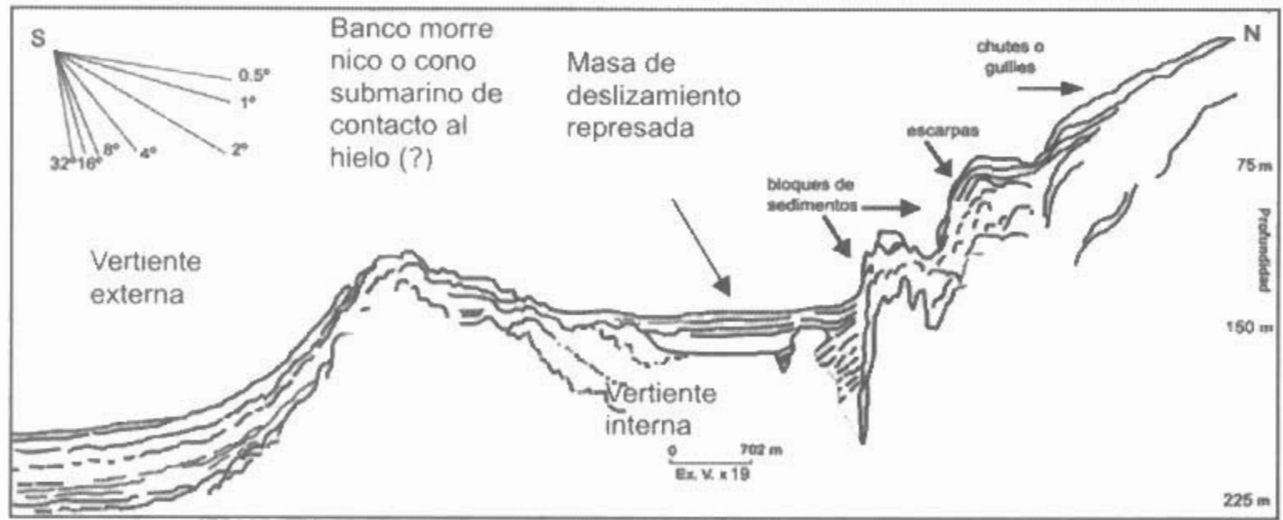

a

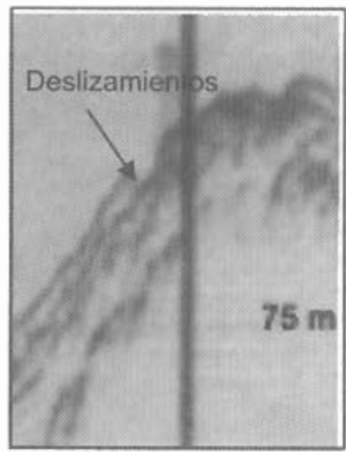

b

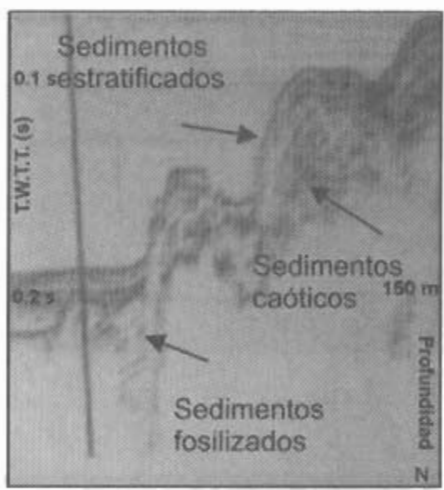

Fig. 8. Fiordo Aysén. Sector proximal. Situación compleja en la zona de delta submarino del río Aysén, donde se mezclan sedimentos de orígenes y de fases distintas de deposición.

Fig. 8. Aysén fjord. Proximal sector. Complex features at river Aysén submarine delta zone, where sediments of distinct origins and deposition phases interact

\section{Distribución de los fenómenos de vertiente}

A partir dos datos obtenidos se puede observar que:

a. Los movimientos en masa se desarrollan con más frecuencia en gradientes desde $1^{\circ}$ hasta $8^{\circ}$ de pendiente, pero también han sido identificados en pendientes inferiores a $1^{\circ}$ (Fig. 9a). El rango de pendiente más activo en fenómenos de vertientes se encuentra entre 2 y $4^{\circ}$ (Fig. 9c), siendo la pendiente de $4^{\circ}$ la más frecuente para estos procesos. $\mathrm{La}$ acumulación de sedimentos se hace más difícil en pendientes superiores a $16^{\circ}$. Las vertientes con pendientes más elevadas se encuentran en el canal Jacaf y fiordo Aysén. b. Los movimientos en masa se efectúan con mayor intensidad entre 50 y $250 \mathrm{~m}$; la profundidad media donde se efectúan los movimientos en masa es de 200 metros, con actividad más frecuente, entre los 100 y $150 \mathrm{~m}$. Los ejemplos en mayores profundidades se encuentran en el fiordo Aysén y canal Jacaf ( Figs. 3 a 8 y 9d).

c. Los deslizamientos tienen acción más efectiva en pendientes entre 2 y $4^{\circ}$, siendo la clase de hasta $1^{\circ}$ también representativa para ese tipo de movimiento en masa. No se verifican deslizamientos en pendientes superiores a $8^{\circ}$ (Fig.9e). Los flujos de detritos se realizan en todas las clases de pendientes, siendo la más activa, entre 4 y $8^{\circ}$ (Fig. 9f). 

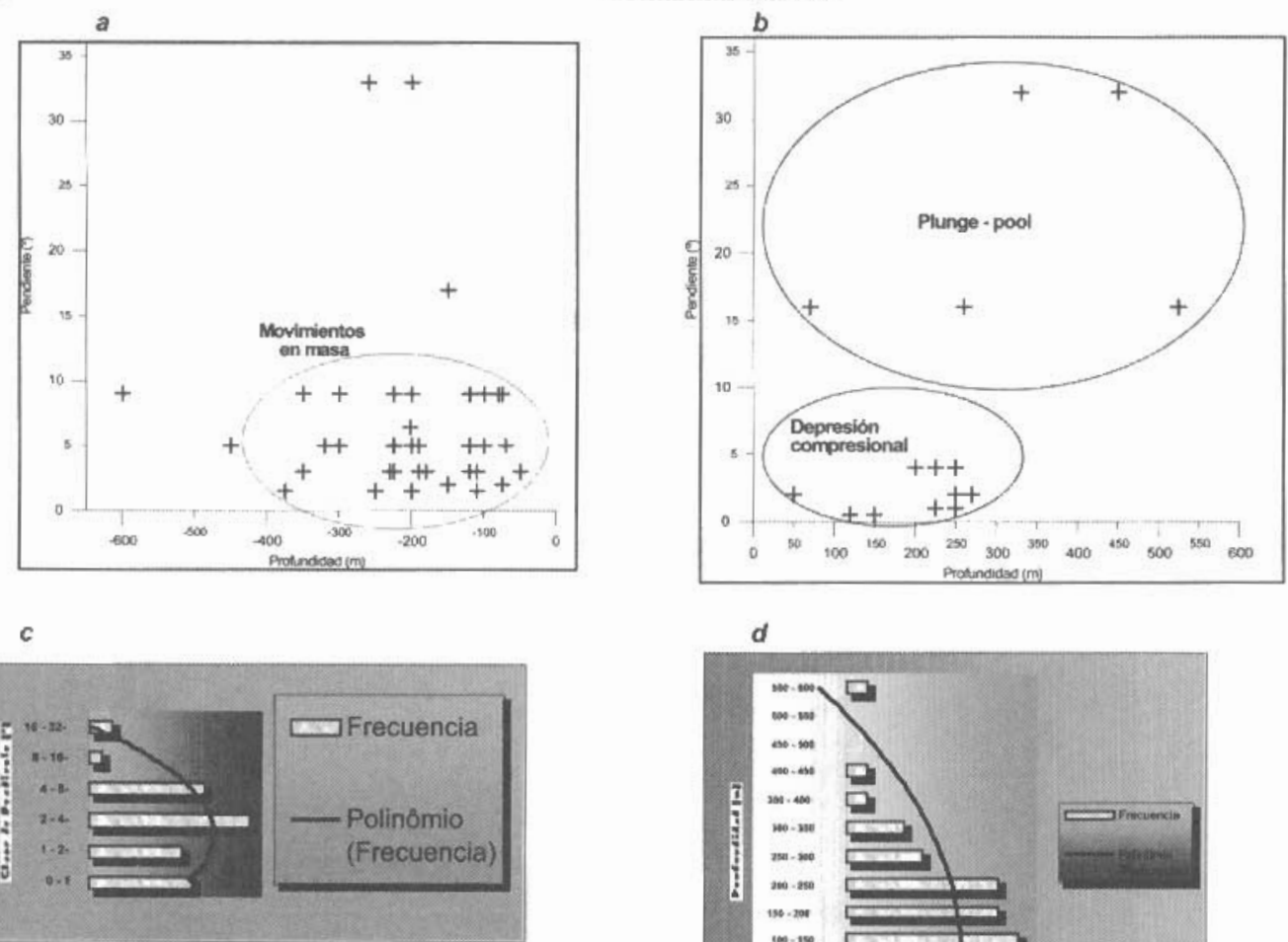

e
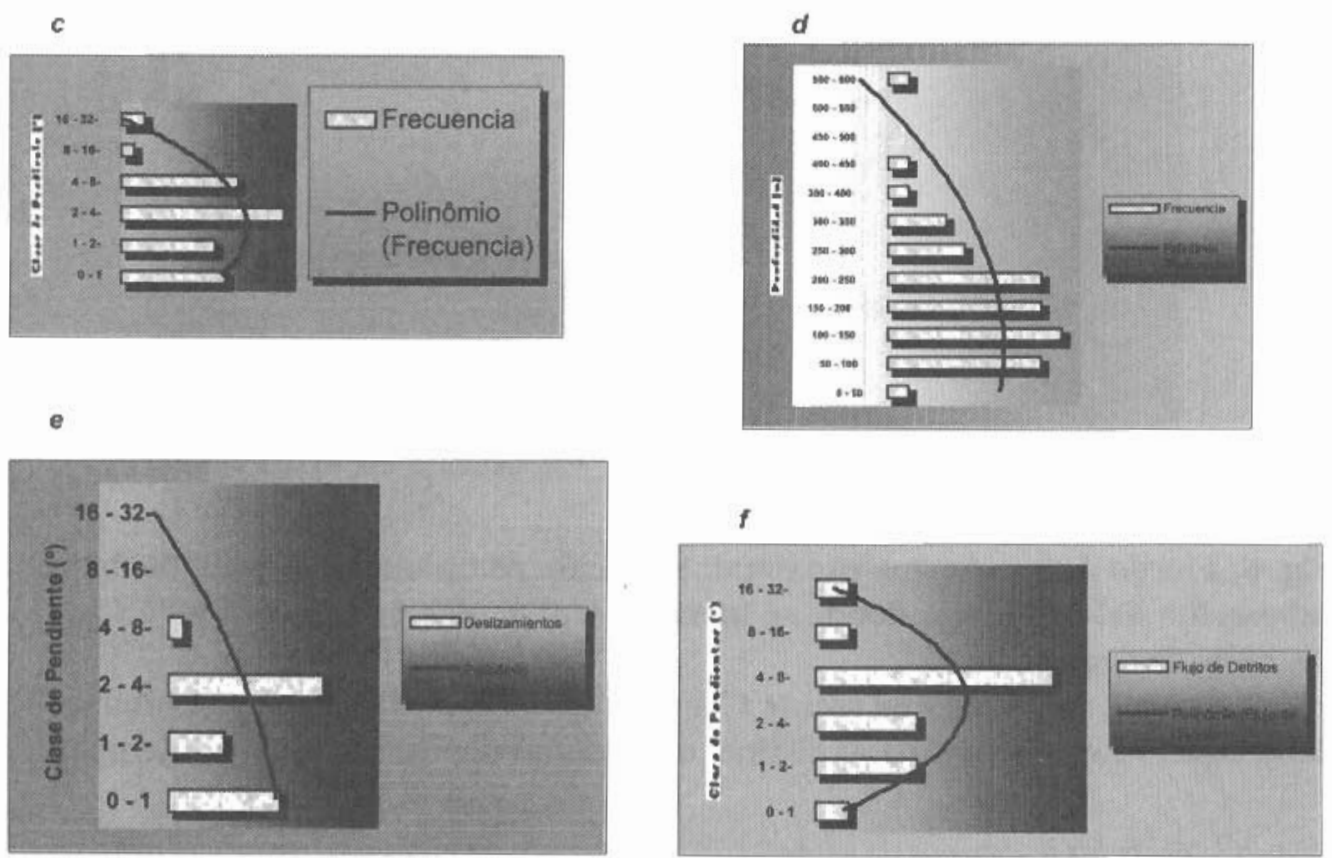

FIG. 9a. Distribución de los movimientos en masa de acuerdo con la pendiente y profundidad.

Fig. 9b. Distribución de plunge - pool y deformación compresional con la pendiente $y$ profundidad.

Fig. 9c. Distribución de los movimientos en masa por clase de pendientes.

Fig. 9d. Distribución de los movimientos en masa por clases de profundidad.

Fig. 9e. Distribución de los deslizamientos por clase de pendiente.

Fig. 9f. Distribución de los flujos de detritos por clase de pendiente.

Fig. 9a. Mass movement distribution according slope and depth.

Fig. 9b. Plunge - pool and compressional deformation distribution according slope and depth.

Fig. 9c. Mass movement distribution according slope class.

Fig. 9d. Mass movement distribution according depth class.

Fig. 9e. Slides distribution according slope class.

Fig. 9f. Debris flows distribution according slope class. 
d. Formas como los plunge pools y las depresiones compresionales se desarrollan en distintos ambientes. Los plunge pools son encontrados, preferentemente, en pendientes sobre $16^{\circ}$ y en las mayores profundidades del canal Jacaf y fiordo Aysén, mientras las depresiones compresionales se forman en pendientes hasta $4^{\circ}$, y no se encuentran en profundidades superiores a los 300 metros (Fig. 9b). La frecuencia de los eventos de plunge pools, no obstante, es inferior al de las depresiones compresionales, lo que es comprensible debido a la cantidad superior de los eventos de deslizamiento.

\section{DISCUSION}

\section{Dinamica de vertientes y depositos de fondo}

\section{Deformaciones asociadas a deslizamientos}

En pendientes suaves, los depósitos formados en la parte inferior de la vertiente reflejan el movimiento originado vertiente arriba. La morfología del fondo de fiordos como Quitralco, Cupquelán y Puyuguapi (Figs. 3b y 5) presenta formas que ARAYA VERGARA (1998) identifica como sedimentos remoldeados y punta de compresión de deslizamiento. Tales formas se complemen$\tan$ con las descritas por SYVITSKI et al. (1987) en fiordos canadienses, como la depresión compresional, ya que próximas a esas últimas se encuentran las puntas de compresión por deslizamiento y los sedimentos remoldeados.

Los sedimentos remoldeados próximos a la base de las vertientes pueden ser el resultado del movimiento realizado en la parte inferior de la masa que sufre deslizamiento. Según DIKAU et al. (1996), quienes analizan los movimientos en masa subaéreos, el desplazamiento de masa sedimentaria y las tensiones producidas por los mismos son responsables por procesos de abombamiento y soterramiento en la parte inferior de la superficie deslizada, lo que puede producir formas redondeadas, pero irregulares (hummocky topography).
Dependiendo del espesor y de la pendiente, el material desplazado sobre la vertiente se extenderá más allá de la misma hacia una potencial zona de compresión, si las capas sedimentarias están asentadas sobre una base rocosa irregular o recubriendo antiguos bancos morrénicos. Ejemplos de dicho proceso son encontrados en los fiordos Puyuguapi y Quitralco y muestran que los sedimentos modernos sufrieron deformaciones debido a los deslizamientos, que los fueron comprimiendo contra una elevación topográfica.

Aún en la parte distal del fiordo Aysén, la forma acuñada de la terraza indica que la zona de erosión se mezcla con zonas de deposición (Fig.3c). La masa sedimentaria desplazada desde la vertiente superior es erosionada en su parte inferior por posibles acciones de corrientes de fondo, dejando expuestos los sedimentos más antiguos representados por tonos acústicos más intensos. Situación similar fue descrita por BARRIER \& PIPER (1982 in SYVITSKI \& SHAW, 1995), en depósitos del Holoceno en Makkovik Bay, Labrador.

En el canal Jacaf, también en su área distal, los deslizamientos de la masa de sedimentos acústicamente semi-transparentes se efectúan en las vertientes a menor profundidad (hasta los $-100 \mathrm{~m}$ ). Por el carácter acústico semitransparente a transparente de los sedimentos del canal Jacaf y fiordo Aysén, debe tratarse de sedimentos finos: arenas y fangos (HOLTEDAHL, 1989; VORREN et al. 1989; SVENDSEN et al. 1992; DOMACK et al. 1994; ANDERSEN et al. 1996; YOON et al. 1997; SYVITSKI et al. 1997; GILBERT et al. 1998; TAYLOR et al. 2000). Según SYVITSKI et al. (1987), esos materiales pueden sufrir remoldelamiento en las capas más superficiales debido a los efectos de la liquefacción a que están sujetos (Fig. 7).

En la zona proximal del fiordo Aysén los bloques de sedimentos de estructura laminada (superficial) y caótica (subsuperficial) que presentan rasgos de intensa deformación, pueden estar asociadas a deslizamientos rotacionales sucesivos. Como se observa en 
la Fig. 8, la deformación de los sedimentos es más acentuada hacia la base de la vertiente que en su parte superior.

Formas como éstas son descritas en ambientes deltaicos submarinos por PRIOR et al. (1981) y KOSTASCHUK \& McCANN (1987). El material deformado es considerado como parte de la zona de acumulación del material transportado desde vertiente arriba. Los rasgos de la parte superior son descritos como chutes o gullies (surcos), en la zona a través de la cual los sedimentos se desplazan.

Las señales de deslizamientos observadas en las porciones medias e inferiores de la vertiente (Fig. 8b) pueden indicar que tal zona sufre inestabilidad debido al suministro de sedimentos desde la parte superior. Según KOSTACHUK \& McCANN (1987), deslizamientos rotacionales son comunes en vertientes medio-inferiores de ambientes de deltas submarinos, pero en el análisis presente estos rasgos no han podido ser identificados.

\section{Deformaciones asociadas a flujos de detritos}

Pese la dificultad de determinar con precisión la categoría de movimiento en masa, los rasgos acústicos asociados principalmente a la estructura interna de la masa sedimentaria (reflectores de fondo y de subfondo de tono medio a elevado [ABAZURRA, 1991] y estructura interna caótica [ELVERHÖI et al. 1983; ELVERHÖI, 1984; KARL, 1989; STOCKER et al. 1997]) pueden indicar deformaciones por flujos de masa (DAMUTH, 1978).

En las vertientes más empinadas del fiordo Aysén y canal Jacaf, la identificación de plunge pools permite la asociación de tales geoformas a posibles flujos de detritos (Fig.7). La generación de plunge pools, formas generalmente asociadas a actividades de avalanchas de detritos en vertientes subaéreas empinadas (LUCKMAN, 1992; BLIKRA \& NEMEC, 1998), ha te- nido poca referencia en ambientes submarinos. Sin embargo, AARSETH et al. (1989) y SEXTON et al. (1992), los identifican en algunos de los fiordos de la costa oeste de Noruega y de Svarbard, respectivamente, y su generación se da en ambientes cuyo desplazamiento de la masa desde la vertiente hacia al fondo se da de una forma muy abrupta.

\section{Relación entre vertientes y fondo en el Canal Jacaf y Seno Ventisquero}

En las cuencas con explanada de represamiento alternadas con acumulaciones caóticas las deformaciones en las vertientes y en el fondo de las cubetas están relacionadas con la dinámica compleja entre los umbrales y las explanadas de represamiento.

Según las descripciones de ARAYA VERGARA (1998), las vertientes multisecuenciales están condicionadas por la superposición de sedimentos. En la zona de contacto entre la acumulación caótica y las capas laminadas en material de la vertiente se engrana con los sedimentos estratificados en forma de cuña.

Por los rasgos acústicos y por la forma de la masa sedimentaria, gran parte de los umbrales en el interior del canal Jacaf puede estar asociada a bancos morrénicos (POWELL, 1981, 1984, 1991; POWELL \& MOLNIA, 1989; POWELL \& ALLEY, 1997; CAI et al. 1997).

La interestratificación entre los materiales de vertiente y del fondo puede ser el resultado de flujos de detritos y deslizamientos. Los sedimentos estratificados sugieren que esos fueron depositados por corrientes de turbidez originadas por los movimientos en masa desde las vertientes, como los descritos por ELVERHÖI (1984), MACKIEVICKZ et al. (1984), SMITH et al. (1991 in COFAIGH et al. 1999), COWAN et al. (1998) y COFAIGH \& DOWDESWELL (2001).

La forma de cuña descrita y nombrada por ARAYA - VERGARA (1998) como lengua 
de material caótico puede estar relacionada con las formas de cuña glacimarina (glacimarine wedge shape, POWELL \& ALLEY 1997). Esas últimas geoformas son consideradas como asociadas al sistema de grounding-line y son comunes en áreas entre bancos morrénicos, donde los flujos de gravedad de las vertientes se mezclan en interlaminaciones (Fig. 6)

\section{Variacion de pendiente y profundidad}

La mayoría de las actividades en las vertientes se produce en pendiente de hasta $8^{\circ}$. Lo mismo ha sido constatado en ambientes de plataformas continentales (BARNES \& LEWIS, 1991; McADOO et al. 2001; McADOO, 2000a y b; LOCAT \& LEE, 2000). En ambiente de fiordos JANSEN $e t$ al. (1987) han identificado deslizamientos en vertientes submarinas de menos de $1^{\circ}$.

En los fiordos de Norpatagonia, aparte de la concentración de los procesos de vertientes en los órdenes de pendiente relatados, se pudo encontrar que - aunque se trate de pendientes suaves - hay posiblemente un ángulo crítico en el cual una variación ya puede causar diferencias de comportamiento. Así, los deslizamientos fueron identificados principalmente en el rango 2 y $4^{\circ}$, mientras que el flujo de detritos, en el rango de 4 y $8^{\circ}$. Depresiones compresionales se encuentran en rangos de pendientes entre 2 y $4^{\circ}$, lo que puede permitir asociar los movimientos de deslizamientos a dichas condiciones.

\section{Causas de los fenomenos de vertientes}

Ha sido abordada la importancia de la variación de la pendiente en la estabilidad de la vertiente, no obstante ese no parece ser el factor preponderante en la determinación de la ruptura de la masa sedimentaria y de su desplazamiento sobre las vertientes submarinas. La complejidad de los movimientos en masa submarina puede ser mayor si son consideradas las varias posibles fases reconocibles en el desarrollo de la vertiente (LOCAT \& LEE, 2000). Los investigadores han especificado muchos factores posibles que inician los movimientos en masa submarinos (ver Materiales y Métodos).

En ambientes de plataforma continental y en muchos fiordos de mundo el factor más actuante para la activación los movimientos en masa submarinos parece relacionarse con actividades sísmicas (LEE et al. 1993 in McADDO et al. 2000a; SYVITSKI \& SCHAFER, 1996; BØE et al. 2000 ). Sin embargo, no se ha hecho todavía tal correlación en fiordos chilenos.

\section{Los fenomenos de vertientes en el contex- to temporal}

Para la evolución de las vertientes y de los fondos de los fiordos se considera, en las cuencas con explanada de represamiento uniforme, dos fases principales (ARAYA VERGARA, 1998): fase inferior y fase superior.

La fase inferior parece tener su relación con la Última Glaciación, documentada por HEUSSER (1990), con efectos submarinos discutidos por ARAYA-VERGARA (1998). La existencia de rasgos postdeposicionales en bancos morrénicos, por ejemplo, indica remoción y redeposición de los sedimentos por deslizamientos y por flujos de detritos que en esa fase no se produjeron mucho más allá de las vertientes. Tales ejemplos y aquellos representados en el canal Jacaf van al encuentro de las observaciones de POWELL (1991), que sugieren que los depósitos de los movimientos en masa son inherentes la mantención de las vertientes del sistema. En éste, las acumulaciones y deformaciones del fondo están más relacionadas con las actividades de corrientes de turbidez.

Las capas superficiales más preservadas disponen de dataciones por Pb210 (SALAMANCA, 1996) y de los cores del Polar Duke (LEVENTER et al. 1995), que pueden ser relacionados a la fase superior. Los datos de $\mathrm{Pb} 210$ son apenas de profundidad decimétrica e indican edades máximas de cientos de años (ver Materiales y Métodos). ARAYA - VERGARA (1998) sugiere, 
con cierto margen de especulación, edades entre 2600 y 7300 años en el interior del fiordo Aysén y entre 1000 y 3000 años en el fiordo Quitralco, asumiendo potencias de sedimentos entre 5 y $14 \mathrm{~m}$ de espesor, respectivamente. Por otro lado, los datos generados por el Polar Duke (LEVENTER et al. 1995) indican edades en escalas milenares para los fondos marinos, siendo las más antiguas en los dominios externos (ARAYA VERGARA, 1997). En un core en el Canal Moraleda, próximo a la salida del fiordo Aysén, la edad aproximada del fondo es $\sim 13000$ años C14.

En ese sentido, se deduce que las estructuras sedimentarias de la fase superior están asociadas a los eventos de transporte y de deposición efectuados en el Holoceno, época en que los fenómenos de vertientes, como los deslizamientos, se observan con más frecuencia en los registros. Por lo tanto, formas como la depresión compresional, la punta de compresión y el abombamiento proximal son rasgos peculiares a los procesos de deformación efectuados a lo largo de la fase superior.

\section{CONCLUSION}

Las secuencias sedimentarias en la morfogénesis identificadas en los registros de perfilador de subfondo $3.5 \mathrm{kHz}$ son resultados de procesos de sedimentación y de erosión que se desarrollaron a partir de la retirada de la masa de hielo desde el Último Máximo Glacial. Esa fase se caracteriza igualmente por la capacidad de los fiordos de capturar y distribuir los sedimentos a lo largo de sus cuencas internas.

La masa sedimentaria movilizada por los varios fenómenos de remoción en masa, ha generado formas entre las que se han ido encontrando nuevas categorías que, comparadas con las encotradas por otros autores, permiten enriquecer la comprensión del sistema de vertientes.

Se observa en las actividades de las vertientes y su relación con el fondo marino un relevante papel de las pendientes, pero otros fac- tores igualmente actúan sobre ellas. La generación de más datos morfométricos debe ser capaz de proveer un mayor conjunto de informaciones sobre el ambiente, los mecanismos de transporte, la estabilidad de la vertiente y la respuesta del fondo a esos procesos.

Para la profundización de estos estudios existen dificultades relacionadas con la accesibilidad y la percepción visual de ambientes submarinos, principalmente los de gran profundidad. Es necesario integrar al levantamiento con perfilador de subfondo el barrido con multi-beam (tipo de sensor del que ya dispone el pais), simulación digital de escenas submarinas tridimensionales (cuya eficiencia se puede ver en Figs. 2 y 3 ) y mejor apoyo de éstos productos al análisis morfométrico.

\section{REFERENCIAS}

ABARRUZA, J., 1991. Sonar de barrido lateral y penetradores de sedimentos. Instituto Hidrográfico de la Marina, Cádiz.

AARSETH, I., 1997. Western Norwegian fjord sediments: age, volume, stratigraphy, and role as temporary depository during glacial cycles. Mar.Geol., 143: 39-53.

ANDERSEN, E.S., T.M. DOKKEN, A. ELVERHØI, A. SOLHEIM \& I. FOSSEN, 1996. Late Quaternary sedimentation and glacial history of the western Svalbard continental margin. Mar. Geol., 133: 123-156.

ARAYA-VERGARA, J.F., 1996. Geomorfología comparada de los fiordos de Chiloé y Aysén. En: Comité Oceanográfico Nacional (Ed.), Resultados Crucero CimarFiordo 1, Resúmenes Ampliados. CONA, Valparaíso, pp. 15-17.

ARAYA - VERGARA, J.F., 1997. Perfiles geomorfológicos de los fiordos y depresión longitudinal de Norpatagonia. Cienc. Tecnol. Mar., 20: 3-22.

ARAYA - VERGARA, J.F., 1998. El problema genético de los fondos de los fiordos 
Norpatagónicos. Invest. Mar. Valparaíso, 26: 71-81.

ARAYA - VERGARA, J.F., 1999a. Secuencia de formas deposicionales en la fractura del Canal Messier, Patagonia Central. Invest. Mar., Valparaíso, 27: 39-52.

ARAYA - VERGARA, J.F., 1999b. Perfiles longitudinales de fiordos de Patagonia Central. Cienc. Tecnol. Mar., 22: 3-29.

ARAYA - VERGARA, J.F., 2000. Perfiles submarinos de los piedmonts del Estrecho de Magallanes y Bahía Nassau, Chile Austral. Anales del Instituto de la Patagonia, Serie Cs. Nat. Chile, 28: 23-40.

BARNES, P. M. \& K.B. LEWIS, 1991. Sheet slides and rotational on a convergent margin: the Kidnappers Slide, New Zealand. Sedimentology, 38: 205-221.

BLIKRA, L.H. \& W. NEMEC, 1998. Postglacial colluvium in Western Norway: depositional processes, facies and paleoclimatic record. Sedimentology, 45: 909-959.

BØE, R. M. HOVLAND, A. INSTANES, L. RISE \& S. VASSHUS, 2000. Submarine slide scars and mass movements in Karmsundet and Skudenesfjorden, southwestern Norway: morphology and evolution. Mar. Geol., 167: 147-165.

CAI, J., R.D. POWELL, E.A. COWAN \& P.R. CARLSON, 1997. Lithofacies and seismic-reflection interpretation of temperate glacimarine sedimentation in Tarr Inlet, Glacier Bay, Alaska. Mar.Geol., 143: 5-37.

CARLSON, P.R., 1989. Seismic reflection characteristics of glacial and glacimarine sediment in the gulf of Alaska and adjacent fjords. Mar. Geol., 85: 391-416.

COFAIGH, C., D.S. LEMMEN, D.J.A. EVANS \& J. BEDNARSKI, 1999. Glacial landform - sediment assemblages in the Canadian High Arctic and their implications for Late Quaternary glaciation. Ann.of Glaciol., 28: 195-208.

COFAIGH, C.O. \& J.A. DOWDESWELL, 2001. Laminated sediments in glacimarine environments: diagnostic criteria for their interpretation. Quatern. Sci. R.., 20: 1411 1436.

COWAN, E.A., J. CAI, R.D. POWELL, K.C.SERAMUR, V.L. SPURGEON, 1998. Modern tidal rhythmites deposited in a deepwater estuary. Geo-Marine Letters 18: 40-48.

DAMUTH, J.E., 1978. Echo character of the Norwegian - Greenland sea: relationship to Quaternary sedimentation. Mar. Geol., 28: 1-36.

DIKAU, R., D. BRUNDSEN, L. SCHROTT, M.L. IBSEN, 1996. Landslide recognition. John Wiley \& Sons, Chichester.

DaSILVA, J.L., J.B. ANDERSON \& J. STRAVERS, 1997. Seismic facies changes along a nearly continuous $24^{\circ}$ latitudinal transect: the fjords of Chile and the northern Antarctic Peninsula. Mar. Geol., 143: 103-123.

DOMACK., E.W., C. McCLENNEN, P. MANLEY \& S. ISHMAN, 1994. Very high resolution stratigraphy of Late Quaternary glacial marine sediments in fjords and offshore basins, Antarctic Peninsula. Terra Antartica, 1(2): 269-270.

ELVERHÖI, A., 1984. Glacigenic and associated marine sediments in the Weddell sea, fjords of Spitsbergen and the Barents sea: a review. Mar. Geol., 57: 53-88.

ELVERHÖI, A., $\emptyset$, LøNNE \& R. SELAND, 1983. Glacimarine sedimentation in a modern fjord environment, Spitsbergen, Polar Res., 1: 127-149.

GILBERT, R., N. NIELSEN, J.R. DESLOGES \& M. RASCH, 1998. Contrasting glacimarine sedimentary environments of two arctic fiords on Disko, West Greenland. Mar. Geol., 147: 63-83. 
HEUSSER, C.J., 1990. Chilotan piedmont glacier in the southern Andes during the last glacial maximum. R. Geol. de Chile, 17: 318.

HOLTEDAHL, H., 1989. Submarine end moraines and associated deposits off the south coast of Norway. Mar. Geol., 88: 23-48.

KARL, H.A., 1989. High-resolution seismicreflection interpretations of some sediment deposits, Antarctic continental margin: focus on the Western Ross Sea. Mar. Geol., 85: 205223.

KOSTASCHUK, R.A. \& S.B. McCANN, 1987. Subaqueous morphology and slope processes in a fjord delta, Bella Coola, British Columbia. Can. J. Earth Sci. 24: 52-59.

KENNETT, J., 1982. Marine Geology. Prentice Hall, New Jersey.

LEVENTER, A., S. AAVANG, J. STRAVERS, S. ISHMAN \& L. KRISSEK, 1995. Holocene sedimentation rates on the southern Chilean continental margin. Antarct. J. U.S., 30 (5): 13-16.

LUCKMAN, B.H., 1992. Debris flow and snow avalanche landforms in the Lairig Ghru, Cairngorm Mountains, Scotland. Geogr. Annaler, 74 A (2-3): 109-121.

McADOO, B., 2000a. Mapping submarine slope failures. vassun.vassar.edu/ brmcadoo/ mapping_slides.htm

McADOO, B., 2000b. Morphometric analysis of submarine landslides. vassun.vassar.edu/ brmcad oo/ landslides_slides.htm

McADOO, B., L.F. PRATSON \& D.L. ORANGE, 2001. Submarine landslide Geomorphology, U.S. Continental Slope. Mar. Geol., 169 (1-2): 103-136.

MACKIEWICKS, N.E., R.D. POWELL, P.R. CARLSON, B.F. MOLNIA, 1984. Interlaminated ice-proximal glacimarine sediments in Muir Inlet, Alaska. Mar. Geol., 57: 113-147.

MULDER, T. \& P. COCHONAT, 1996. Classification of offshore mass movements. J. Sedim. Res., 66 (1): 43-57.

NIEMEYER, H.R., J.M. SKARMETA, R.P. FUENZALIDA \& W.N. ESPINOSA, 1984. Carta Geológica de Chile. Hojas Península de Taitao y Puerto Aysén. Servicio Nacional de Geología y Minería.

PRIETO, M.J., G. ERCILLA, M. CANALS \& M. BATIST, 1999. Seismic stratigraphy of the Central Bransfield Basin (NW Antarctic Peninsula). Interpretation of deposits and sedimentary processes in a glacio-marine environment. Mar. Geol., 157: 47-68.

POSTMA, G., 1986, Clasification for sediment gravity-flow deposits based on flow conditions during sedimentation. Geology, $14: 291-294$

POWELL, R.D.,1981. A model for sedimentation by tidewater glaciers. Ann. Glaciol., 2: 129-134.

POWELL, R.D., 1983. Glacial-marine sedimentation processes and lithofacies of temperate tidewater glaciers, Glacier Bay, Alaska. In: MOLNIA, B.F: (Ed.). GlacialMarine Sedimentation, Plenum Press, New York.

POWELL, R.D., 1984. Glacimarine processes and inductive' 'ithofacies modelling of ice shelf and tidewaier glacier sediments based on quaternary examples. Mar. Geol., 57: $1-52$.

POWELL, R.D., 1988. Advances of glacial tidewater fronts in Glacier Bay, Alaska. In: MILNER A.M. \& J.D. WOOD (Eds.), Proceedings of the Second Glacier Bay System Symposium, Glacier Bay, Alaska, 1988. U.S. Department of Interior, National Park Service, U.S. Government Printing Office: $67-73$. 
POWELL, R.D., 1990. Glacimarine processes at grounding-line fans and their growth to ice-contact deltas. In: DOWDESWELL, J.A. \& SCOURCE, J.D. (Eds), Glacimarine Environments: Processes and Sediments. Geological Society Special Publication $\mathrm{N}^{\circ}$ 53: 53-73.

POWELL, R.D., 1991. Grounding-line systems as second-order controls on fluctuations of tidewater termini of temperate glaciers. Geological Society of America, Special Paper, 261: 75-93.

POWELL, R.D. \& B.F. MOLNIA, 1989. Glacimarine sedimentary processes facies and morphology of the south-southeast Alaska shelf and fjords. Mar.Geol., 85: 359-390.

POWELL, R.D. \& R.B. ALLEY, 1997. Grounding-line systems: processes, glaciological inferences and the stratigraphic record. In: Geology and Seismic Stratigraphy of the Antarctic Margin, Part 2. Antarctic Res. Series, 71: 169-187.

SALAMANCA, M.A.O., 1996. Geocronología de sedimentos marinos de la zona de fiordos de la XI Región. En: Comité Oceanográfico Nacional (Ed.), Resultados Crucero Cimar-Fiordo 1, Resúmenes Ampliados. CONA, Valparaíso, pp. 64-68.

SEXTON, D.J., J.A. DOWDESWELL, A. SOLHEIM \& A. ELVERHØI, 1992. Seismic architecture and sedimentation in northwest Spitsbergen fjords. Mar. Geol.,103: 53-68.

STOKER, M.S., J.B. PHEASANT \& H. JOSENHANS, 1997. Seismic Methods and Interpretation in DAVIEW, T.A. et al (Eds.) Glaciated Continental Margins: An Atlas of Acoustic Imagery, Chapman \& Hall.

SVENDSEN, J.I., J. MANGERUD, A. ELVERHØI, A. SOLHEIM \& R.T.E. SCHÜTTENHELM, 1992. The Late Weichselian glacial maximum on western Spitsbergen inferred from offshore sediment cores. Mar. Geol., 104: 1-7.
SYVITSKI, J.P.M., D.C. BURREL \& J.M. SKER, 1987. FJORDS, Processes and Products. Springer-Verlag, New York, 379pp.

SYVITSKI, J.P.M. \& D.B. PRAEG, 1989. Quaternary sedimentation in the St. Lawrence estuary and adjoining areas, Eastern Canada: an overview based on high-resolution seismostratigraphy. Géogr. Phys. Quat. 43, 291-310.

SYVITSKI, J.P.M. \& J. SHAW, 1995. Sedimentology and geomorphology of fjords. In PERILLO, G.M.E. (ed.). Geomorphology and sedimentology of estuaries. Development in Sedimentology, 53: 113-178.

SYVITSKI, J.P.M. \& C.T. SCHAFER, 1996. Evidence for an earthquake-triggered basin collapse in Saguenay Fjord, Canada. Sedimentary Geol., 104: 127-153.

SYVITSKI, J.P.M. \& H.J. LEE, 1997. Postglacial sequence stratigraphy of Lake Melville, Labrador. Mar. Geol., 143: 55-79.

TAYLOR, J., J.A. DOWDESWELL, N.H. KENYON, R.J. WHITTINGTON, Tj.C.E. VAN WEERING \& J. MIENERT, 2000. Morphology and Late Quaternary sedimentation on the North Faeroes slope and abyssal plain, North Atlantic. Mar. Geol., 168: 1-24.

VORREN, T.O., E. LEBESBYE, K. ANDREASSEN \& K.B. LARSEN, 1989. Glacigenic sediments on a passive continental margin as exemplified by the Barents Sea. Mar. Geol., 85: 251-272.

YOON, H.I., M.W. HAN, B.K. PARK, J.K. OH \& S.K. CHANG, 1997. Glacimarine sedimentation and paleo-glacial setting of Maxwell Bay and its tributary embayment, Marian Cove, South Shetland Islands, West Antarctica. Mar. Geol., 140: 265-282.

YOUNG, A., 1975. Slopes. Longman, London. 


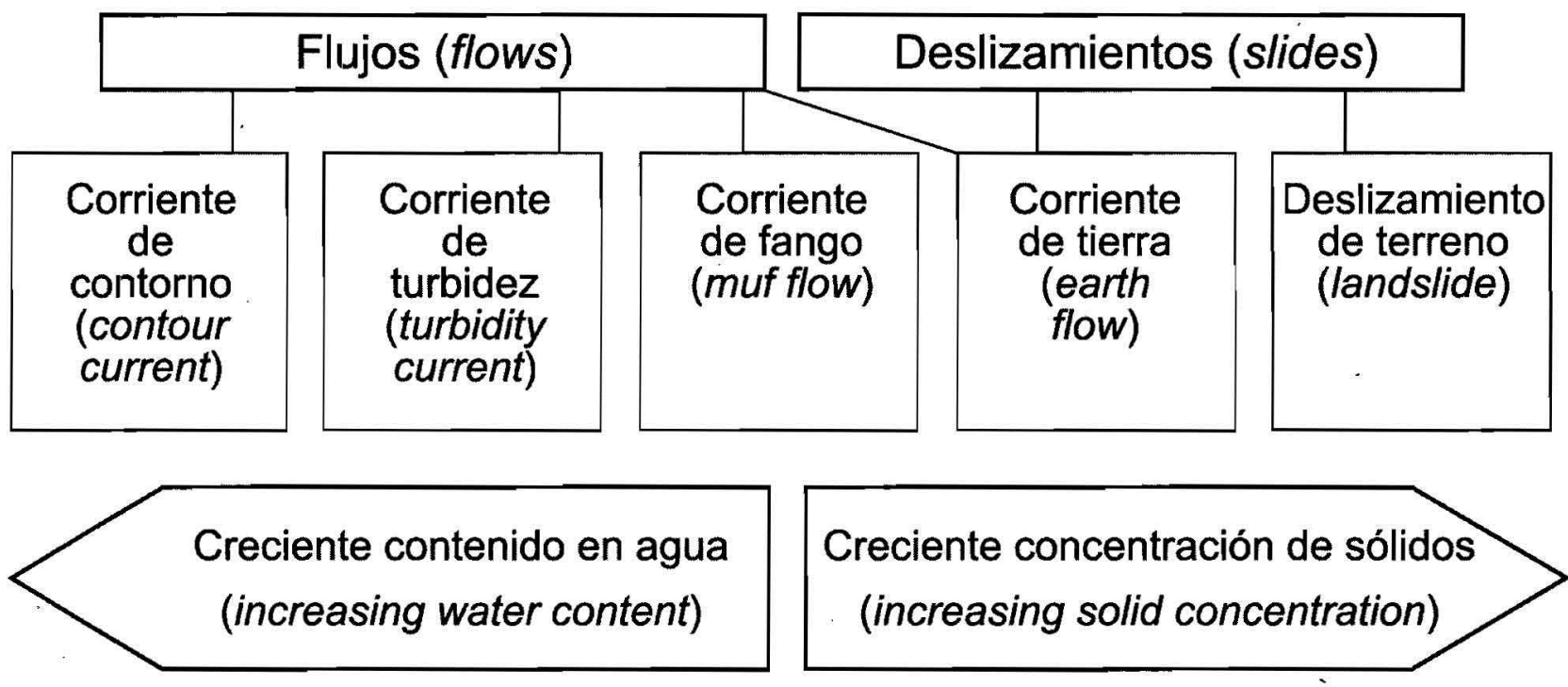

Dinámica de vertientes en paisajes submarinos (seascapes): Categorías principales de movimientos en masa submarinos identificados en canales y fiordos patagónicos, clasificados según los criterios de Mulder \& Cochonat (1996, J. Sedim. Res., 66(1): 43-67), Meunier (1993, modif. por Locat \& Lee, 2000, Proc. Internat. Symp. Landslides, Cardiff: 1 -30) y Araya-Vergara (1998, Invest. Mar. Valp., 26: 71-81) 